\title{
Enrichment of anaerobic ammonium oxidation (anammox) bacteria for short start-up of the anammox process: a review
}

\begin{abstract}
The application of the anaerobic ammonium oxidation (anammox) reaction in a biological nitrogen removal system to treat wastewater has become of great interest since its discovery. The anammox reaction is performed by anammox bacteria that belong to the Planctomycete phylum. The reaction occurs in the presence of ammonium using nitrite as the substrate under anaerobic conditions. However, the bacteria have an extremely slow growth rate and stringent metabolic conditions that cause difficulty in culturing and applying the system for wastewater treatment. Anammox enrichment has a long start-up period for the anammox process that hinders researchers using laboratory and full-scale systems for the first time. Many attempts have been made to culture anammox to establish a successful anammox culture with a shorter start-up period for the anammox reaction and high nitrogen removal activity. This paper reviews previous studies on anammox enrichment with emphasis on (i) inoculum selection, (ii) enrichment techniques and (iii) factors influencing anammox enrichment. This review will assist researchers in planning and designing an appropriate anammox enrichment. The findings should widen the application of anammox in biological nitrogen removal systems for nitrogenous wastewater.
\end{abstract}

Keyword: Anaerobic ammonium oxidation (anammox); Biological nitrogen removal; Enrichment; Nitrogenous wastewater; Start-up 